\title{
Long-term Outcome of Chilblains Associated with SARS-CoV-2
}

\author{
Florence POIZEAU ${ }^{1,2}$, Sébastien BARBAROT ${ }^{3}$, Yannick LE CORRE ${ }^{4}$, Emilie BRENAUT'5 ${ }^{5}$ Mahtab SAMIMI ${ }^{6}$, Hélène AUBERT ${ }^{3}$, \\ Alexis TOUBEL 2 and Alain DUPUY ${ }^{1,2}$ \\ ${ }^{1}$ EA 7449 REPERES (Pharmacoepidemiology and Health Services Research), Rennes 1 University, Department of Dermatology; ${ }^{2}$ CHU Rennes, \\ Rennes, ${ }^{3} \mathrm{CHU}$ Nantes, Nantes, ${ }^{4} \mathrm{CHU}$ Angers, Angers, ${ }^{5} \mathrm{CHU}$ Brest, Brest and ${ }^{6} \mathrm{CHU}$ Tours, Tours, France
}

\begin{abstract}
Numerous cases of chilblains have been observed in the course if the COVID-19 pandemic. The aims of this study were to provide comprehensive follow-up data for patients reporting chilblains, and to determine the risk factors for incomplete recovery. Patients referred to $\mathbf{5}$ hospitals in France between March and May 2020 for chilblains were surveyed on December 2020. A teleconsultation was offered. Among 82 patients reporting chilblains, 27 (33\%) reported complete recovery, 33 $(40 \%)$ had recurrences of chilblains after their hands and feet had returned to normal, and 22 (27\%) developed persistent acral manifestations, mostly acrocyanosis, with or without further recurrences of chilblains. Most recurrences of chilblains occurred during the following autumn and winter. A past history of chilblains was not associated with recurrences or persistent acral manifestations. Women had a significantly higher risk of developing recurrences or persistent acral manifestations (odds ratio 1.30; $95 \%$ confidence interval 1.06-1.59). In conclusion, two-thirds of patients reporting chilblains at the start of the COVID-19 pandemic experienced persistent or recurrent acral manifestations after a 10-month follow-up.
\end{abstract}

Key words: chilblains; pernio; perniosis; acral manifestation; SARS-CoV-2; COVID-19; recurrence.

Acta Derm Venereol 2021; 101: adv00614.

Accepted Sep 13, 2021; Epub ahead of print Sep 13, 2021

Corr: Alain Dupuy, Department of Dermatology, CHU Rennes, 2 rue Henri le Guilloux, FR-35000 Rennes, France. E-mail: alain.dupuy@chu-rennes.fr

$\mathrm{N}$ umerous cases of chilblains were reported throughout Europe and North America during the early months of the COVID-19 pandemic (1-9). The association of these acral manifestations with SARS-CoV-2 infection has been a subject of debate, since most patients did not have virological confirmation of SARS-CoV-2 infection. It has been hypothesized that a strong antiviral type I interferon response could account for rapid viral clearance, explaining the low levels of anti-SARSCoV-2 antibodies. This immune response could also be responsible for interferon-induced skin lesions resembling chilblains, as seen in genetic interferonopathies $(8,10-13)$. The outcome of cutaneous manifestations related to SARS-CoV-2 infection is currently arousing increasing interest (14-20). The current study provides a 10-12-month follow-up of patients with chilblains who were included in a previous case-control study (21). A

\section{SIGNIFICANCE}

Reports on the long-term outcome of patients with chilblains associated with SARS-CoV-2 are rare and fragmentary. This study provides comprehensive follow-up data for 82 patients who reported chilblains during spring 2020, at the beginning of the COVID-19 pandemic in France. Two-thirds of patients reported recurrences of chilblains or persistence of acral manifestations over a 10-12-month follow-up. Recurrences of chilblains mostly occurred during autumn and winter 2020, suggesting that either weather conditions or further contact with SARSCoV-2 could act as new triggers. Women had a higher risk of experiencing incomplete recovery.

further aim of this study was to determine the risk factors associated with incomplete recovery.

\section{MATERIALS AND METHODS}

\section{Participants and information collection}

Individuals referred for chilblains during the first lockdown due to COVID-19 (17 March to 11 May 2020) in Western France were included in a case-control study exploring the aetiological role of SARS-CoV-2 exposure (21). They were referred to 5 University Hospitals (Rennes, Brest, Nantes, Angers and Tours) and all diagnoses were validated by a dermatologist as being chilblains, with the presence of localized erythema and swelling involving acral sites, persistent for more than $24 \mathrm{~h}$. Information on these chilblains was collected as part of the case-control study. The current study aimed to describe the follow-up from the onset of the reported chilblains (between 31 January and 3 May 2020) to an end date (between 31 December 2020 and 4 February 2021), according to patients. An online questionnaire was sent to patients on 31 December 2020, and patients with incomplete recovery from the chilblains were offered a teleconsultation in January 2021 to collect detailed clinical and virological information. This information was further updated between 29 January and 4 February 2021.

\section{Statistical analysis}

Continuous variables were described using means and standard deviation (SD) or medians and range. Categorical variables were described using frequencies (\%). The median duration of chilblain recurrences with $95 \%$ confidence interval (CI) was computed using the Kaplan-Meier method (data censored for ongoing chilblains between 29 January and 4 February 2021). A univariate logistic regression analysis was used to assess the association between incomplete recovery (i.e. persistent or recurrent acral manifestations) and patient characteristics or features related to the initial flare of chilblains. Type 1 error was set at $5 \%$. For patients who experienced persistent or recurrent acral manifestations and had a teleconsultation, a time-line graph was plotted covering the period 
from 29 January 2020 to 29 January 2021. Statistical analyses and graphical plot were performed using RStudio Version 1.0.136 (RStudio Inc., Boston, MA, USA).

\section{RESULTS}

\section{Study participants}

Of the 102 individuals with chilblains who participated in the case-control study (21), 82 (80\%) gave information on follow-up via a questionnaire. The 55 patients who reported persistent acral manifestations or recurrence of chilblains were solicited for a teleconsultation and 43 accepted. The study flowchart is shown in Fig. 1.

\section{Overview of the follow-up: 3 phases}

To provide a synthetic description of the follow-up of patients who reported chilblains during the first lockdown resulting from the COVID-19 pandemic, 3 successive periods were defined diagrammatically: $(i)$ a first phase during spring 2020 (depicted as the inner ring in Fig. 2), corresponding to chilblains reported during the first wave of the COVID-19 pandemic; (ii) a second phase during summer 2020 (depicted as the intermediate ring in Fig. 2), following resolution of the chilblains. Most patients recovered; however, a proportion of patient developed chronic acral manifestations distinct from chilblains; (iii) a third phase, roughly during the autumn and the early winter months of 2020 to 2021 (depicted as the outer ring in Fig. 2), during which recurrences of chilblains were observed.

First phase (spring 2020). The clinical characteristics of the 82 patients who reported chilblains during the first lockdown (17 March to 11 May 2020) are shown in Table I.

Second phase (summer 2020). All 82 patients reported resolution of their chilblains. For a majority of patients $(n=60)$, their hands and feet returned to normal. However, in 22 patients $(27 \%)$, the symptoms evolved towards persistent acral manifestations, distinct from chilblains,

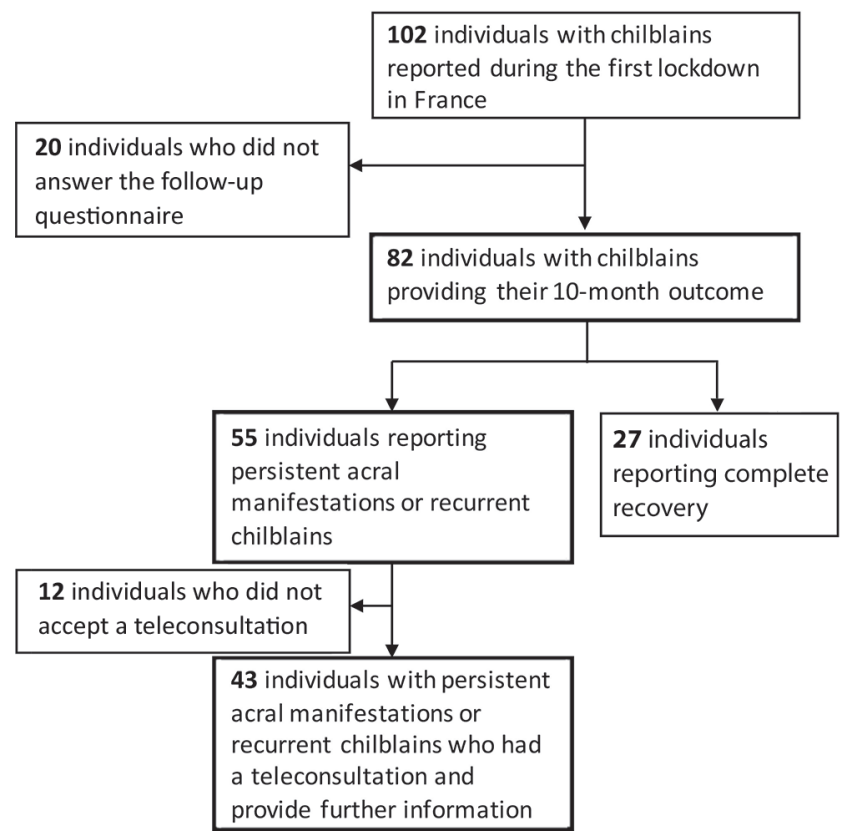

Fig. 1. Study flowchart.

including acrocyanosis $(n=14)$, erythromelalgia $(n=4)$, Raynaud phenomenon $(n=3)$, with toe oedema and stiffness in 1 case, and cold toes $(n=1)$.

Third phase (autumn and winter 2020/2021). A total of 46 patients $(56 \%)$ had recurrent chilblains after 10 months of follow-up (Fig. 2). Examples of persistent acral manifestations associated with chilblain recurrences are shown in Fig. 3. Most chilblain recurrences $(n=38$, $83 \%$ ) occurred after 1 October 2020. Patients reported $2(n=36)$ or $3(n=10)$ flares of chilblains. Recurrences were less, equally or more severe than the first flare in $32(70 \%), 7(15 \%)$ and $7(15 \%)$ cases, respectively. The lesions were located on the feet, the hands or both in 34 (74\%), 7 (15\%), and 5 (11\%) cases, respectively. Regarding the context of chilblain recurrences, $6(13 \%)$ occurred after acknowledged exposure to cold. Five patients $(11 \%)$ reported possible viral symptoms (influenza-like illness, diarrhoea, headache) during the recurrences, and

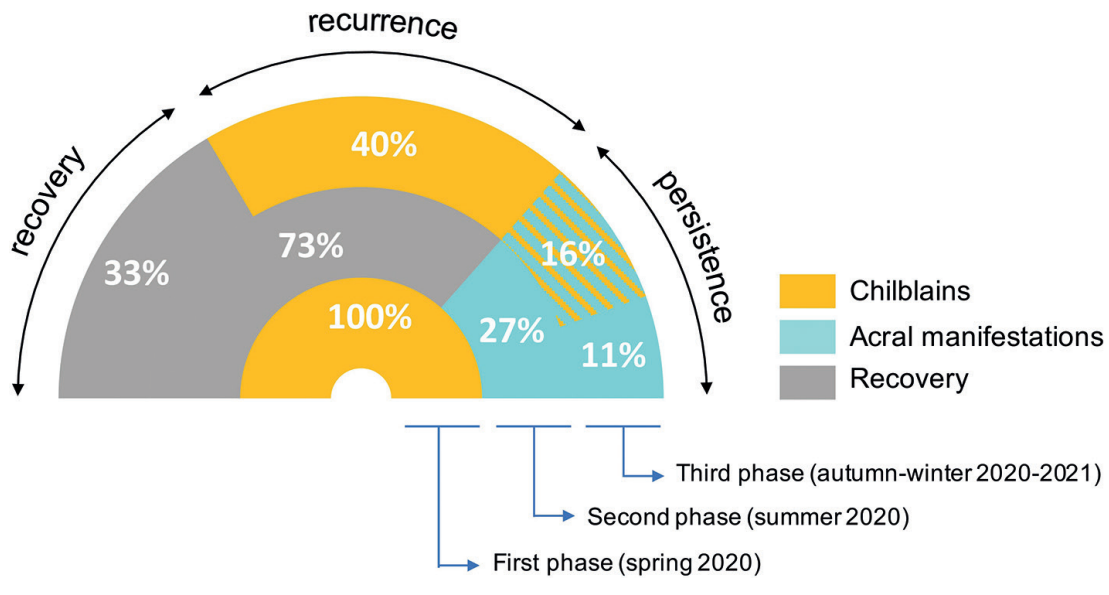

Fig. 2. Diagrammatic sequence of acral manifestations during follow-up. Among patients with chilblains during the first lockdown in spring 2020 ( $n=82(100 \%)$; inner ring of the doughnut chart), chilblains resolved in all cases. During the following weeks (intermediate ring - summer), hands and feet returned to normal $(n=60(73 \%))$ or evolved towards persistent acral manifestations $(n=22(27 \%)$, including acrocyanosis, erythromelalgia, Raynaud phenomenon, toe oedema and stiffness, and cold toes). After 10-12 months of follow-up (outer ring - mostly in the autumn and winter), 27 (33\%) patients remained free of lesions, 46 (56\%) had chilblain recurrences (including $33(40 \%)$ patients whose hands and feet had returned to normal), and $9(11 \%)$ had persistent acral manifestations without recurrence of chilblains. This description enables 3 evolution profiles to be identified (recovery, recurrence or persistence). 
Table I. Characteristics of patients reporting chilblains during the first lockdown

\begin{tabular}{ll}
\hline Characteristics & Patients $(n=82)$ \\
\hline Age in years, mean (SD) & $30.4(14.9)$ \\
Sex, women, $n(\%)$ & $49(59.8)$ \\
History of chilblains, $n(\%)$ & $16(19.5)$ \\
History of Raynaud syndrome, $n(\%)$ & $16(19.5)$ \\
SARS-CoV-2 serology, $n(\%)$ & \\
Positive & $3(4.9)$ \\
Negative & $58(95.1)$ \\
Localization of chilblains, $n(\%)$ & \\
Feet & $59(72.0)$ \\
Hands & $13(15.9)$ \\
Both & $10(12.2)$ \\
Symptoms suggesting COVID-19,$n(\%)$ & $28(34.1)$ \\
Duration of chilblains in days, mean (SD) & $33.7(24.6)$
\end{tabular}

${ }^{a}$ Among anosmia, ageusia, fever, asthenia, rhinitis, sore throat, cough, dyspnoea. SD: standard deviation.

2 patients underwent rt-PCR testing for SARS-CoV-2 with a negative result.

\section{Three evolution profiles: recovery, chilblain recurrences and persistent acral manifestations}

From this synthetic description of the follow-up in 82 patients, 3 evolution profiles can be distinguished among patients having reported chilblains during the first lockdown of the COVID-19 pandemic. In the first group $(n=27,33 \%)$, the patients recovered from their chilblains with no recurrence observed within the study time-frame ("recovery" profile); the second group $(n=33,40 \%)$ gathered patients who recovered from their chilblains in the spring, but experienced chilblain recurrences in the autumn/winter ("recurrence" profile); the third group $(n=22,27 \%)$ gathered patients who, after their chilblains in the spring, presented persistent acral manifestations throughout the study period, with or without chilblain recurrences ("persistence" profile). These 3 profiles are depicted in Fig. 2. Fig. 4 presents on a time-line the individual follow-up of the 43 patients with a "recurrence" or "persistence" profile for whom detailed information was collected by teleconsultation.

\section{Characteristics of patients with chilblain recurrences or persistent acral manifestations}

Among the $67 \%$ of patients who developed chilblain recurrences or persistent acral manifestations (i.e. "recurrence" and "persistence" profiles), the median age was 29 years (range $12-84$ years), and 38 patients (69\%) were women. Complete information regarding medical history and clinical manifestations was obtained from 43 patients (Fig. 1). Five had a history of autoimmune disease, including thyroiditis $(n=3)$, ulcerative colitis $(n=1)$ and Sjögren's syndrome $(n=1)$. Two patients had inflammatory arthralgia of the knees, wrists and elbows at the time of their chilblain recurrence. Six patients reported persistent extra-dermatological manifestations within the study time-frame: $4(9 \%)$ had a new onset of sicca syndrome, 1 patient had haematuria and leukocyturia, and 1 reported persistent diarrhoea (Fig. 4).

\section{Risk factors for chilblain recurrences or persistent acral manifestations}

Risk factors for incomplete recovery following chilblains (i.e., a "recurrence" or "persistence" profile) were sought, including age, sex, history of chilblains, previous Raynaud syndrome, positive anti-SARS-CoV-2 antibodies, systemic COVID-19 symptoms, localization and duration of the first flare of chilblains. Women had a significantly higher risk of developing recurrences or persistent acral manifestations (OR 1.30; 95\% CI 1.06-1.59). Neither a past history of chilblains (OR 1.02; 95\% CI 0.79-1.32), nor any other characteristics were associated with chilblain recurrences or persistent acral manifestations (Table II).

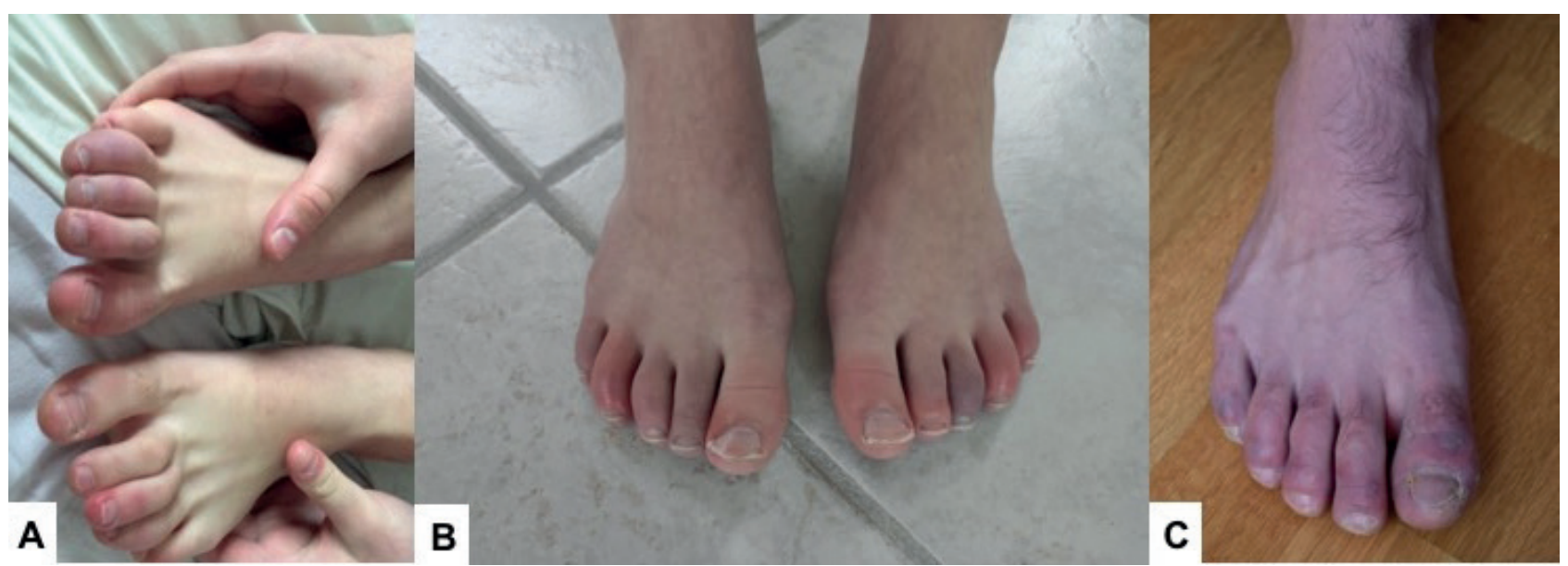

Fig. 3. Examples of patients with persistent acral manifestations who experienced chilblain recurrences. (A, B) Distal erythema of multiple toes with some oedema in a symmetrical pattern, leaving persistent cyanotic discoloration (patient numbers 3 and 35 ). (C) Violaceous and erythematous papules and patches on the toes (patient number 29). Patient numbers are reported in Fig. 4. 
Chilblains on feet - Chilblains on hands + History of chilblains

Chilblains on feet and hands

- Persistent acral manifestations

- Persistent systemic symptoms

๑ Positive serology

- Negative serology

OExposure to cold $\triangle$ Positive rt-PCR testing

$\Delta$ Negative rt-PCR testing

$\rightarrow$ Onset of suspected viral symptoms

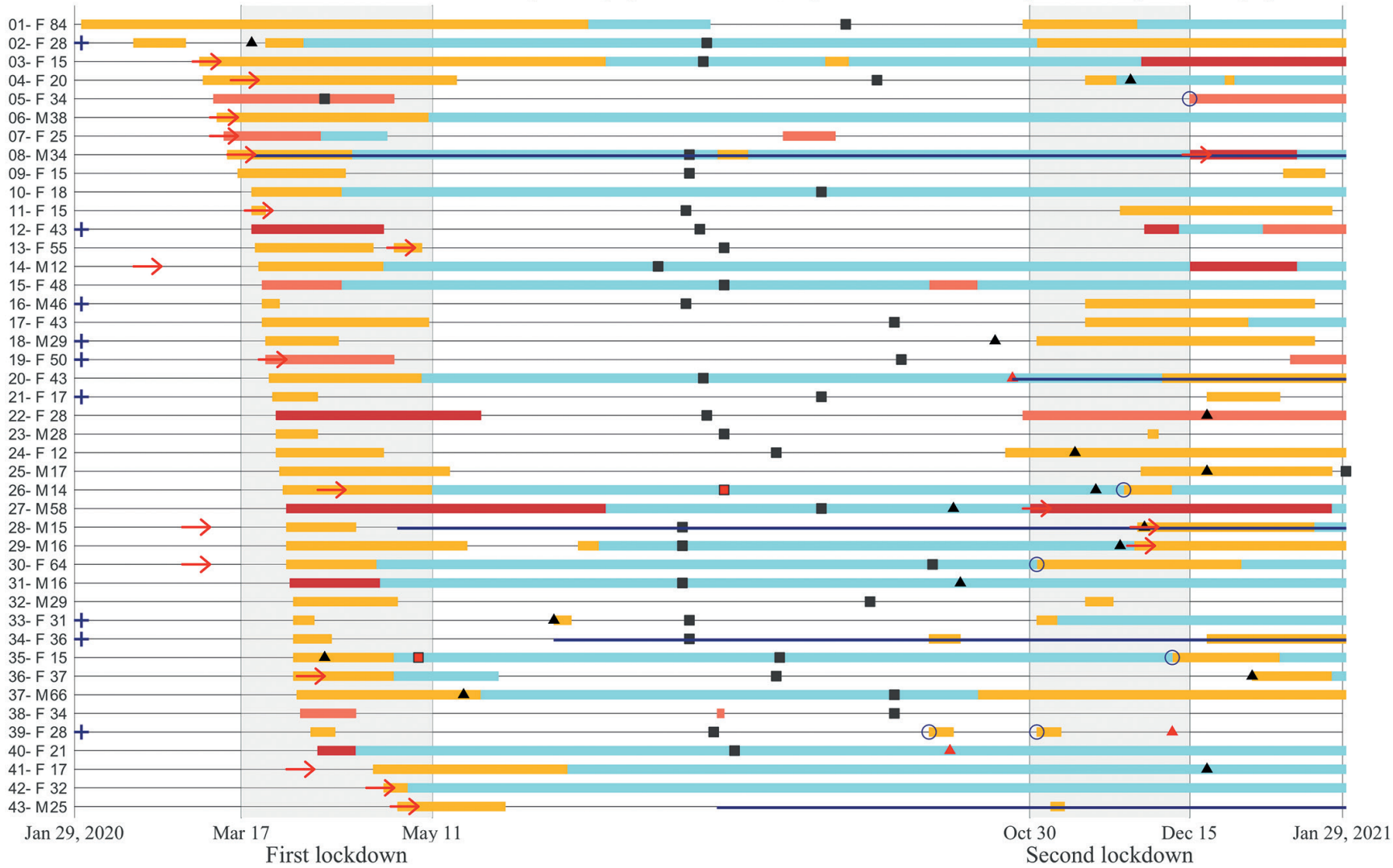

First lockdown

Second lockdown

Fig. 4. Time-line plot showing the chronology of clinical manifestations over 12 months for patients reporting persistent or recurrent acral manifestations (patients with a "persistence" or "recurrence" profile). Sex (F: female; M: male) and age (years) are specified at the start of the line for each patient reported. Persistent systemic manifestations were as follow: patient numbers 8 , 20, 34, and 43 reported sicca syndrome, associated with a persistent hyposmia in 1 patient (patient number 20). Patient number 42 had haematuria and leukocyturia associated with chilblains, which receded over 7 months. Patient number 28 reported diarrhoea after his first flare of chilblains (normal abdominal ultrasound, normal stool test for bacteria and parasites), which persisted thereafter.

\section{DISCUSSION}

Among the 82 patients who had chilblains during the first lockdown (21), 33\% recovered without further acral manifestations over a 10-month period. In contrast, $40 \%$ had chilblain recurrences after initial recovery, mostly during autumn and winter, and $27 \%$ had persistent acral manifestations from the summer following the chilblains

Table II. Risk factors for persistent or recurrent acral manifestations following chilblains

\begin{tabular}{llll}
\hline & & & $p-$ \\
& OR & $95 \%$ CI & \begin{tabular}{l}
$p$ value \\
\hline Age, for 1 year
\end{tabular} \\
Sex, for women & 1.003 & $0.996-1.010$ & 0.41 \\
History of chilblains & 1.3 & $1.06-1.59$ & 0.01 \\
History of Raynaud syndrome & 1.02 & $0.79-1.32$ & 0.88 \\
Seropositivity for SARS-CoV-2 & 1.02 & $0.79-1.32$ & 0.88 \\
Duration of the initial flare of chilblains, for 1 day & 0.99 & $0.57-1.73$ & 0.98 \\
Symptoms possibly related to COVID-19 & 1.003 & $0.999-1.007$ & 0.20 \\
Localization of chilblains (ref=hands) & 1.07 & $0.86-1.33$ & 0.55 \\
Feet & & & 0.15 \\
Feet and hands & 1.31 & $0.99-1.73$ & \\
\hline
\end{tabular}

aserological tests performed between May and August 2020. ${ }^{\mathrm{b}} \mathrm{Among}$ anosmia, ageusia, fever, asthenia, rhinitis, sore throat, cough, dyspnoea.

OR: odds ratio; $95 \%$ CI: $95 \%$ confidence interval. (acrocyanosis, erythromelalgia, Raynaud phenomenon, toe oedema and stiffness, and cold toes), with or without additional chilblain recurrences. Female sex was associated with chilblain recurrences or persistent acral manifestations.

Numerous series of patients with chilblains were reported during the early months of the COVID-19 pandemic in various countries (1-9). Follow-up data are important because idiopathic chilblains and acral manifestations usually develop in chronic or recurrent mode (22). With nearly 1 year of follow-up, our estimation of $56 \%$ of patients experiencing chilblain recurrences is in line with the proportion recently suggested $(19,20)$. Even assuming that all $20 \%$ of the patients who did not answer the follow-up questionnaire recovered, the percentage of patients who experienced chilblain recurrences would still amount to $45 \%$. Previous studies reported only $3-18 \%$ of recurrences (15-17), but provided very fragmentary follow-up data. In addition, the percentage of chilblain recurrences could also depend on exposure to other triggers (e.g. cold and wet weather), which vary across different geographical areas. Percentages are also 
liable to change with increasing follow-up duration, since patients classified as having a "recovery" profile could experience chilblain recurrences later on.

Our diagrammatic presentation based on 3 phases (in the spring at the onset of the COVID-19 pandemic, the following summer, and the following autumn/winter), and 3 evolution profiles (recovery, recurrence or persistence) has the advantage of synthetizing follow-up information. However, not all individuals fit perfectly into this pattern (for instance, some patients experienced chilblain recurrences during the summer), but a time-line graph with individualized information highlights both the exceptions and the common characteristics. The most salient common characteristic lies in the temporal clustering of chilblain recurrences after 1 October 2020. Because they occurred in a colder and damper period in Western France, and also at the time when there was a "second wave" of intense viral circulation in the country, leading to a second national lockdown, no unequivocal interpretation can be made. Both exposure to weather conditions and increased exposure to SARS-CoV-2 could be the answers to the "why now?" question for this temporal clustering of chilblain recurrences. Regarding the conditions specifically associated with lockdown (e.g. conditions leading to a certain type of viral exposure within households), they may have had a role, but their specific contribution could not be analysed in the case-control study (21). In addition, the current study was limited to the follow-up of chilblains that occurred during the first national lockdown in France, and new cases were not collected at the time of the second lockdown. Data regarding ex novo occurrences of chilblains are scarce, which could be explained either by a truly low incidence or by under-reporting (16-18). Furthermore, it would have been interesting to evaluate the COVID-19 vaccines as a potential trigger for acral manifestations in these patients (23). However, at the time of evaluation, COVID-19 vaccines were not widely available.

The term "chilblains" (or "pernio") is advocated to refer to what were previously called "chilblains-like" lesions, because, in all series $(8,9,24,25)$, they do not differ from classic chilblains on clinical and histological grounds (22). The specificity can be seen in the causal relationship between SARS-CoV-2 and chilblains (21, 26). Classic chilblains occur as recurrent episodes, generally after exposure to cold and wet conditions, which exemplifies how exposure can trigger specific clinical manifestations in predisposed individuals (not everybody develops chilblains when exposed to cold). In the current study, $20 \%$ of patients had a past history of chilblains and $80 \%$ experienced a flare of chilblains for the first time during the COVID-19 pandemic. We suggest that, in individuals with a past history of chilblains, exposure to SARS-CoV-2 in confined homes could act as a new trigger, and in others, as a sufficient cause. The identification of an infectious trigger for chilblains could even lead to the suggestion that other seasonal viruses, possibly human coronaviruses, have a role in triggering seasonal chilblains.

A notable result of the current follow-up study is that approximately one-quarter of the patients who had chilblains during the first lockdown evolved towards a variety of acral manifestations during the summer season. Although it is well-known that some people have acral manifestations all year round, the development of persistent acrocyanosis following chilblains is unusual (22). Pathophysiologically, the more substantiated hypothesis to link exposure to SARS-CoV-2 and chilblains relates to a strong antiviral interferon immune response $(8,13$, 27). In medical circumstances when abnormally high interferon levels are reached, acral manifestations have been observed: chilblains are one of the hallmarks of genetic type I interferonopathies (28), and acrocyanosis as well as Raynaud phenomenon have been reported following treatment with interferon- $\alpha(29,30)$. Persistent acral manifestations could be promoted by a long-lasting interferon-driven immunological response, which could also account for other manifestations, possibly related to auto-immunity, as observed in some of our patients. Whether these persistent or recurrent acral manifestations, sometimes accompanied by extra-dermatological manifestations, belong to the "long COVID" spectrum is an open question waiting for a better understanding of the pathophysiology of post-COVID syndromes (15, 19, 31-35).

This study summarizes the different evolution profiles among patients who had chilblains during the first lockdown of the COVID-19 pandemic in France, and highlights persistent and recurrent acral manifestations. The underlying biological and immunological substratum remains to be investigated. These acral manifestations require follow-up, with a specific attention to extradermatological manifestations, and auto-immunity in particular.

\section{ACKNOWLEDGEMENTS}

Angela Verdier, MA, and Sarah Leyshon, MA, SARL L'Auracoise, revised the article for the English language. They received compensation for their contribution.

The study was approved by the CHU Rennes Ethics Committee on 24 June, 2020 (reference 20.79).

Conflicts of interest. MS received reimbursement for travel and/ or accommodation expenses for attending medical meetings from Bristol Myers Squibb, Lilly, Galderma International, Janssen, Abbvie, MSD, outside the submitted work. AD received reimbursement for travel and/or accommodation expenses for attending medical meetings from Sanofi, and personal fees from Sanofi and Leo Pharma, outside the submitted work.

\section{REFERENCES}

1. Recalcati S, Barbagallo T, Frasin LA, Prestinari F, Cogliardi A, Provero MC, et al. Acral cutaneous lesions in the time of CO- 
VID-19. J Eur Acad Dermatol Venereol 2020; 34: e346-347.

2. Freeman EE, McMahon DE, Lipoff JB, Rosenbach M, Kovarik C, Takeshita J, et al. Pernio-like skin lesions associated with COVID-19: a case series of 318 patients from 8 countries. J Am Acad Dermatol 2020; 83: 486-492.

3. Le Cleach L, Dousset L, Assier H, Fourati S, Barbarot S, Boulard C, et al. Most chilblains observed during the COVID-19 outbreak occur in patients who are negative for COVID-19 on polymerase chain reaction and serology testing. $\mathrm{Br} \mathrm{J}$ Dermatol 2020; 183: 866-874.

4. Fernandez-Nieto D, Jimenez-Cauhe J, Suarez-Valle A, Moreno-Arrones OM, Saceda-Corralo D, Arana-Raja A, et al. Characterization of acute acral skin lesions in nonhospitalized patients: a case series of 132 patients during the COVID-19 outbreak. J Am Acad Dermatol 2020; 83: e61-e63.

5. de Masson A, Bouaziz J-D, Sulimovic L, Cassius C, Jachiet M, Ionescu $M-A$, et al. Chilblains is a common cutaneous finding during the COVID-19 pandemic: a retrospective nationwide study from France. J Am Acad Dermatol 2020; 83: 667-670.

6. Galván Casas C, Català A, Carretero Hernández G, RodríguezJiménez P, Fernández-Nieto D, Rodríguez-Villa Lario A, et al. Classification of the cutaneous manifestations of COVID-19: a rapid prospective nationwide consensus study in Spain with 375 cases. Br J Dermatol 2020; 183: 71-77.

7. Piccolo V, Neri I, Filippeschi C, Oranges T, Argenziano G, Battarra VC, et al. Chilblain-like lesions during COVID-19 epidemic: a preliminary study on 63 patients. J Eur Acad Dermatol Venereol 2020; 34: e291-293.

8. Hubiche T, Cardot-Leccia N, Le Duff F, Seitz-Polski B, Giordana $P$, Chiaverini $C$, et al. Clinical, laboratory, and interferonalpha response characteristics of patients with chilblain-like lesions during the COVID-19 pandemic. JAMA Dermatol 2021; 157: 202-206.

9. Herman A, Peeters C, Verroken A, Tromme I, Tennstedt D, Marot $L$, et al. Evaluation of chilblains as a manifestation of the COVID-19 pandemic. JAMA Dermatol 2020; 156: 998-1003.

10. Bessis D. Impaired type I interferon response in SARS-CoV-2 infection: looking through the cutaneous window. $\mathrm{Br} \mathrm{J}$ Dermatol 2021; 184: 11-12.

11. Magro CM, Mulvey JJ, Laurence J, Sanders S, Crowson AN, Grossman $M$, et al. The differing pathophysiologies that underlie COVID-19-associated perniosis and thrombotic retiform purpura: a case series. Br J Dermatol 2021; 184: 141-150.

12. Lipsker D. Paraviral eruptions in the era of COVID-19: do some skin manifestations point to a natural resistance to SARS-CoV-2? Clin Dermatol 2020; 38: 757-761.

13. Damsky W, Peterson D, King B. When interferon tiptoes through COVID-19: pernio-like lesions and their prognostic implications during SARS-CoV-2 infection. J Am Acad Dermatol 2020; 83: e269-e270.

14. Maanaoui S, Salez F, Carpentier O. Recurrence of chilblains during a second contact with SARS-CoV-2: a case report. $\mathrm{Br}$ J Dermatol 2021; 185: 227-228.

15. MCMahon DE, Gallman AE, Hruza GJ, Rosenbach M, Lipoff JB, Desai SR, et al. Long COVID in the skin: a registry analysis of COVID-19 dermatological duration. Lancet Infect Dis 2021; 21: 313-314.

16. Freeman EE, McMahon DE, Lipoff JB, Rosenbach M, Desai SR, Fassett $M$, et al. Cold and COVID: recurrent pernio during the COVID-19 pandemic. Br J Dermatol 2021; 185: 214-216.

17. Signa S, Sementa AR, Coccia MC, Pastorino C, Viglizzo G, Viola $S$, et al. Recurrence of previous chilblain lesions during the second wave of COVID-19: can we still doubt the correlation with SARS-CoV-2? J Eur Acad Dermatol Venereol
2021; 35: e475-e477.

18. Recalcati S, Barbagallo T, Tonolo S, Milani M, Fantini F. Relapse of chilblain-like lesions during the second wave of coronavirus disease 19. J Eur Acad Dermatol Venereol 2021; 35: e315-e316.

19. Moghadam $P$, Frumholtz $L$, Jaume $L$, De Masson $A$, Jachiet $M$, Begon $E$, et al. Frequency of relapse and persistent cutaneous symptoms after a first episode of chilblain-like lesion during the COVID-19 pandemic. J Eur Acad Dermatol Venereol 2021; 35: e566-e568.

20. Hubiche T, Le Duff F, Fontas E, Rapp J, Chiaverini C, Passeron T. Relapse of chilblain-like lesions during the second wave of the COVID-19 pandemic: a cohort follow-up. Br J Dermatol 2021; 185: 858-859.

21. Poizeau F, Oger E, Barbarot S, Le Corre Y, Samimi M, Brenaut $E$, et al. Chilblains during lockdown are associated with household exposure to SARS-CoV-2. A multicentre case-control study. Clin Microbiol Infect 2021 Oct 4 [Online ahead of print].

22. Creamer D. Reactions to cold. In: Burns T, Breathnach S, Cox N, Griffiths C, editors. Rook's textbook of dermatology. Eighth edn. Wiley-Blackwell, Chichester, West Sussex, UK, 2010: p. 28.65-67.

23. McMahon DE, Amerson E, Rosenbach M, Lipoff JB, Moustafa $D$, Tyagi A, et al. Cutaneous reactions reported after Moderna and Pfizer COVID-19 vaccination: a registry-based study of 414 cases. J Am Acad Dermatol 2021; 85: 46-55.

24. Kanitakis J, Lesort C, Danset M, Jullien D. Chilblain-like acral lesions during the COVID-19 pandemic ("COVID toes"): histologic, immunofluorescence, and immunohistochemical study of 17 cases. J Am Acad Dermatol 2020; 83: 870-875.

25. Aschoff R, Zimmermann N, Beissert S, Günther C. Type I interferon signature in chilblain-like lesions associated with the COVID-19 pandemic. Dermatopathol 2020; 7: 57-63.

26. Ortega-Quijano D, Fernandez-Nieto D, Jimenez-Cauhe J, Cortes-Cuevas JL, Marcos-Mencia D, Rodriguez-Dominguez $M$, et al. Association between COVID-19 and chilblains: a case-control study. J Eur Acad Dermatol Venereol 2021; 35: e359-e361.

27. Jabalameli N, Rajabi F, Firooz A, Rezaei N. The overlap between genetic susceptibility to COVID-19 and skin diseases. Immunol Invest 2021 Jan 26 [Epub ahead of print].

28. Volpi S, Picco P, Caorsi R, Candotti F, Gattorno M. Type I interferonopathies in pediatric rheumatology. Pediatr Rheumatol Online J 2016; 14: 35.

29. Campo-Voegeli A, Estrach T, Marti RM, Corominas N, Tuset M, Mascaró JM. Acrocyanosis induced by interferon alpha(2a). Dermatology 1998; 196: 361-363.

30. Kruit WH, Eggermont AM, Stoter G. Interferon-alpha induced Raynaud's syndrome. Ann Oncol 2000; 11: 1501-1502.

31. Carfi A, Bernabei R, Landi F, Gemelli Against COVID-19 PostAcute Care Study Group. Persistent symptoms in patients after acute COVID-19. JAMA 2020; 324: 603-605.

32. Petersen MS, Kristiansen MF, Hanusson KD, Danielsen ME, á Steig B, Gaini S, et al. Long COVID in the Faroe Islands: a longitudinal study among nonhospitalized patients. Clin Infect Dis 2021; 73: e4058-e4063.

33. Logue JK, Franko NM, McCulloch DJ, McDonald D, Magedson $A$, Wolf $C R$, et al. Sequelae in adults at 6 months after COVID-19 infection. JAMA Netw Open 2021; 4: e210830.

34. Salmon-Ceron D, Slama D, De Broucker T, Karmochkine M, Pavie J, Sorbets E, et al. Clinical, virological and imaging profile in patients with prolonged forms of COVID-19: a cross-sectional study. J Infect 2021; 82: e1-4.

35. Nasserie T, Hittle M, Goodman SN. Assessment of the frequency and variety of persistent symptoms among patients with COVID-19. JAMA Netw Open 2021; 4: e2111417. 